\title{
Estudio comparativo de taninos de tara, mimosa y pino como recurtientes.
}

\author{
Hourdebaigt, R..$^{(1)}$, Iade, J. ${ }^{(1)}$, Le Rose, C. ${ }^{(1)}$, Damonte, D. ${ }^{(1)}$ \\ Contacto: rhourde@latu.org.uy \\ (1) Departamento de Cueros - Laboratorio Tecnológico del Uruguay (LATU)
}

\begin{abstract}
Resumen
Los taninos vegetales son productos naturales que tienen la capacidad de formar complejos con proteínas. Dentro de este contexto, son los productos naturales más importantes usados industrialmente en los procesos que transforman las pieles en cueros.

En este proyecto caracterizaremos los taninos utilizados (evaluación de su poder curtiente) y partiendo de una formulación base en función del artículo final elegido (capellada de calzado), se realiza el curtido e iremos variando el curtiente vegetal y evaluando su comportamiento con distintos ensayos.

Los taninos evaluados son mimosa, pino radiata y polvo de tara.

También presentamos una caracterización de los cueros, mostrando los ensayos a realizar a los diferentes tipos de cuero en función de su uso, las normas que aplican para los análisis y especificaciones que nos orienten para los mismos.

Estas especificaciones indican las propiedades que el cuero debe cumplir para que el mismo tenga un comportamiento adecuado durante su fabricación y uso.

Palabras claves: taninos, polifenoles, curtido, recurtido, norma, capellada, forro, suela.
\end{abstract}

\begin{abstract}
The vegetable tannins are natural products that are able to form complex with proteins. Within this context, are the most important natural products used in industrial processes that transform raw hides into leathers.

In this project we wil characterize the tannins used (evaluation of their power tanning), starting with a basic formulation depending on the final article chosen (upper shoes), the process of tanning is carried out varying the vegetable tannin and evaluating its behaviour with different essays.

The evaluated tannins are: mimosa, radiata pine and tare powder. We also present a leather characterization, showing the essays to carry out, the different types of leather depending on its use, the standars used for the analysis and specifications that guide us to them. These specifications indicate the properties that the leather must have to have appropiate behaviour during their manufacture and use. Keywords: tannins, polyphenols, tanning, standar, upper shoes, lining, sole.
\end{abstract}

\section{Introducción}

L os taninos vegetales son productos naturales de peso molecular relativamente alto que tienen la capacidad de formar complejos con los carbohidratos y proteínas. Dentro de este contexto, son de los productos naturales más importantes usados industrialmente, específicamente en los procesos que transforman las pieles en cueros.

Dentro de los artículos fabricados por curtido o recurtido vegetal se pueden citar suela, capellada, plantilla y forro para calzado, cueros para tapicería, para marroquinería entre otros.

Los taninos vegetales se clasifican en dos grupos importantes: los taninos hidrolizables y los condensados.

En virtud de esta importancia es que evaluaremos estas nuevas fuentes de taninos como curtientes y recurtientes.

\section{Características de los taninos}

\section{Extractos curtientes comerciales}

Entre los extractos curtientes comerciales encontramos:

a) extracto de mimosa, fácilmente soluble en agua, da cueros flexibles de color beige amarillento

b) extracto de quebracho natural, da cueros firmes, solubles en frío por bisulfitación da cueros más flexibles y suaves.

c) extracto de castaño, de astringencia elevada, da cueros firmes de color avellana. Este extracto es el más sólido a la luz

d) extracto de castaño, de astringencia elevada, da cueros firmes de color avellana. Este extracto es el más sólido a la luz

e) de yalomea, de gran astringencia da cueros de color amarillento bastante impermeables f) de zumaque, es un extracto suave que penetra rápidamente en la piel, da cuero de tacto suave y flexible y de color muy claro

g) extracto de pino, de gran astringencia, da al cuero un color rojizo.

h) extractos de lignina, en el tratamiento de maderas con sulfitos y bisulfitos para la obtención de la pasta del papel se logran grandes cantidades de compuestos lignosulfónicos solubles que luego son purificadas con tratamientos químicos y desecadas por atomización. Los ácidos lignosulfónicos se fijan bien sobre el colágeno pero no tienen propiedades curtientes, se aplican como auxiliares retardando la fijación del tanino, facilitando la dispersión de los sedimentos y mejorando su difusión en los taninos

\section{Curtientes hidrolizables y condensados}

Los taninos no son idénticos en todos los vegetales, ellos difieren en cuanto a su composición y a sus propiedades químicas especiales según el género botánico donde se encuentren. Son compuestos fenólicos, es decir que su principal función química está representada por el oxidrilo o hidroxilo $\mathrm{OH}$ unido a un núcleo bencénico y que poseen un carácter ácido débil. Los taninos están constituidos por grandes moléculas cuyas soluciones acuosas son coloidales y tendrán tendencia a enturbiarse (flocular) y dar precipitados. La clasificación más acertada es la que la propuesta por Freudenberg en 1920 y que considera dos grupos:

\section{Taninos hidrolizables.}

Los extractos tánicos hidrolizables o pirogálicos son aquellos que por hidrólisisenmedioácidoyaebulliciónformanproductos solublesenagua. 
Su constitución está caracterizada por el hecho de que el núcleo bencénico está unido al segundo compuesto por intermedio de átomos de oxígeno. Depositan, habitualmente, ácido elágico (compuesto amarillento, cristalizado y poco soluble en agua) finamente dividido que forma borra en el fondo de las cubas y eflorescencias en el cuero.

Con sales de hierro dan coloración negro-azulada.

Los extractos tánicos hidrolizables se pueden clasificar en dos grandes grupos:

a) Aquellos que forman ácido gálico y glucosa a través de hidrólisis, llamados extractos gálicos

b) Aquellos otros que dan ácido elágico y glucosa llamados extractos elágicos.

2.1.1.2 Taninos condensados. Los extractos condensados o catequínicos que en las mismas condiciones forman precipitados. Sus núcleos constituyentes están reunidos entre sí con intervención de átomos de carbono. Se los llama catequínicos porque sometidos a destilación seca, casi todos, dan pirocatequína.

\section{Caracterización de cueros y sus normas:}

A continuación se detallan los ensayos a realizar a los diferentes tipos de cuero en función de su uso, las normas que aplican para los análisis y especificaciones para los mismos en nuestro caso vinculado al calzado.

Estos valores son una recopilación de distintos organismos y empresas más importantes del ramo, siendo en última instancia una relación comercial que estima los estándares de calidad de las materias primas para la industria del calzado, prendas y tapicería.

El organismo que regula las normas de cueros es la IULTCS (Unión Internacional de Sociedades de Técnicos del Cuero) entidad internacional que esta formada por todas las asociaciones que corresponden a los diferentes países socios.

La denominación con la sigla IUP se refiere a ensayos físicos, las que lleva la sigla IUC a ensayos químicos y las IUF a solídeces.

Se realizo un recurtido con destino a capellada de calzado, hasta el estado semiterminado, (comúnmente llamado crust, cuero curtido, recurtido, engrasado teñido o no y acondicionado para el acabado).

Adjuntamos normas y especificaciones a los cueros que se utilizaran para suela, capellada y forro.

Estas espécificaciones son a titulo orientativo sobre los estándares de calidad exigidos en este caso para suela, capellada y forro.

\section{Cueros para suela:}

\begin{tabular}{|l|c|l|}
\hline \multicolumn{1}{|c|}{ Ensayo } & Norma & \multicolumn{1}{c|}{ Especificación } \\
\hline Absorción de agua & $\begin{array}{c}\text { IUP 7 - } \\
\text { DIN 53330 }\end{array}$ & $\begin{array}{l}\text { Max. Abs. de agua 2 } \\
\text { horas = 35\% } \\
\text { Max. Abs. de agua 24 } \\
\text { horas = 45\% }\end{array}$ \\
\hline $\begin{array}{l}\text { Permeabilidad al } \\
\text { vapor de agua }\end{array}$ & IUP 15- & Min 53333 $200 \mathrm{mg} / \mathrm{cm}^{2} \mathrm{~h}$ \\
\hline Espesor & IUP 4 & 2,5-5,0 mm \\
\hline pH & IUC 11 & Mín 3 \\
\hline $\begin{array}{l}\text { Tiempo de } \\
\text { penetración } \\
\text { de agua en } \\
\text { condiciones } \\
\text { dinámicas }\end{array}$ & IUP 11 & $\begin{array}{l}\text { Tiempo mín 30 minutos } \\
\text { min. 30 \% }\end{array}$ \\
\hline $\begin{array}{l}\text { Materias totales } \\
\text { solubles en agua }\end{array}$ & IUC 6 & Max. Base seca 20 \% \\
\hline $\begin{array}{l}\text { Cenizas sulfatadas } \\
\text { solubles en agua }\end{array}$ & IUC 6 & $\begin{array}{l}\text { Cenizas solubles max } \\
3 \%\end{array}$ \\
\hline
\end{tabular}

\subsubsection{Cuero para capellada:}

\begin{tabular}{|c|c|c|c|c|}
\hline Ensayo & Norma & \multicolumn{3}{|c|}{ Especificación } \\
\hline $\begin{array}{l}\text { Resistencia a la } \\
\text { flexión }\end{array}$ & $\begin{array}{l}\text { IUP } 39 \\
\text { IUP } 20\end{array}$ & \multicolumn{3}{|c|}{$\begin{array}{l}\text { Cuero acabado: seco } 50000 \text { ciclos } \\
\text { húmedo } 20000 \text { ciclos }\end{array}$} \\
\hline $\begin{array}{l}\text { Tracción y } \\
\text { alargamiento }\end{array}$ & IUP 6 & \multicolumn{2}{|c|}{$\begin{array}{l}\text { Carga }\left(\mathrm{N} / \mathrm{mm}^{2}\right) \\
\text { Alargamiento }\end{array}$} & $55 \%$ \\
\hline $\begin{array}{l}\text { Resistencia al } \\
\text { desgarro }\end{array}$ & IUP 8 & \multicolumn{3}{|c|}{$\begin{array}{l}\text { Calzado con forro } 35 \mathrm{~N} \\
\text { calzado sin forro } 50 \mathrm{~N}\end{array}$} \\
\hline \multirow{2}{*}{$\begin{array}{l}\text { Adhesión a la } \\
\text { terminación } \\
\qquad(\mathrm{N} / 10 \mathrm{~mm})\end{array}$} & \multirow[b]{2}{*}{ IUF 470} & \multirow{2}{*}{$\begin{array}{l}\text { Plena flor } \\
\text { Flor } \\
\text { corregida }\end{array}$} & seco & húmedo \\
\hline & & & $\begin{array}{l}3 \\
5\end{array}$ & $\begin{array}{l}2 \\
3\end{array}$ \\
\hline Espesor & IUP 4 & \multicolumn{3}{|c|}{1,4 a $1,6 \mathrm{~mm}$} \\
\hline $\begin{array}{l}\text { Comportamiento } \\
\text { frente a la } \\
\text { temperatura }\end{array}$ & IUF 458 & \multicolumn{3}{|c|}{$80^{\circ} \mathrm{C}$} \\
\hline $\begin{array}{l}\text { Solidez del } \\
\text { acabado a la luz }\end{array}$ & $\begin{array}{l}\text { IUF } 401 \text { y } \\
\text { IUF } 402\end{array}$ & \multicolumn{3}{|c|}{ Ensayo opcional } \\
\hline $\begin{array}{l}\text { Distensión } \\
\text { rotura de flor } \\
\text { (lastometro) }\end{array}$ & IUP 9 & \multicolumn{3}{|c|}{ Mín 7 mm } \\
\hline Ensayo & Norma & \multicolumn{3}{|c|}{ Especificación } \\
\hline $\begin{array}{l}\text { Solidez de la } \\
\text { terminación } \\
\text { frente a frotes }\end{array}$ & $\begin{array}{l}\text { IUF 450, } \\
\text { DIN } \\
53339\end{array}$ & \multicolumn{3}{|c|}{$\begin{array}{l}\text { Cuero seco, fieltro seco } 50 \text { ciclos } \\
\text { cuero seco, fieltro húmedo } 50 \\
\text { ciclos }\end{array}$} \\
\hline $\begin{array}{l}\text { Solidez a la gota } \\
\text { de agua }\end{array}$ & IUF 420 & \multicolumn{3}{|c|}{ pasa } \\
\hline $\begin{array}{l}\text { Permeabilidad al } \\
\text { vapor de agua }\end{array}$ & IUP 15 & \multicolumn{3}{|c|}{ Mín 200 - 250 mg/cm2/h } \\
\hline $\begin{array}{l}\text { Impermeabilidad } \\
\text { al agua }\end{array}$ & IUP 10 & \multicolumn{3}{|c|}{$\begin{array}{l}\text { Tiempo de penetración } 120 \\
\text { minutos }\end{array}$} \\
\hline $\begin{array}{l}\text { Materias } \\
\text { extraíbles en } \\
\text { cloruro de } \\
\text { metileno }\end{array}$ & IUC 4 & \multicolumn{3}{|c|}{$\begin{array}{l}\text { Hasta } 9 \% \text { para aplicar adhesivo } 1 \\
\text { componente, hasta } 14 \% \text { adhesivo } \\
\text { especial de poliuretano, hasta } 7 \% \\
\text { se puede vulcanizar, hasta } 15 \% \text { se } \\
\text { puede aplicar el procedimiento de } \\
\text { inyección de PVC. } \\
\text { Estos valores dependen de la } \\
\text { distribución de la grasa en el } \\
\text { espesor del cuero y de la clase de } \\
\text { grasa }\end{array}$} \\
\hline
\end{tabular}

\section{Cueros para forro}

\begin{tabular}{|l|c|c|}
\hline \multicolumn{1}{|c|}{ Ensayo } & Norma & \multicolumn{1}{c|}{ Especificación } \\
\hline Solidez al frote & $\begin{array}{c}\text { IUF 450 } \\
\text { (número de } \\
\text { ciclos) }\end{array}$ & $\begin{array}{l}\text { Cuero seco, fieltro seco 100 } \\
\text { Cuero seco fieltro húmedo 50 } \\
\text { Cuero húmedo 50 } \\
\text { fieltro mín 4 (escala grises) }\end{array}$ \\
\hline Solidez al agua & IUF 421 & \multicolumn{1}{|c|}{ Mín 3 } \\
\hline $\begin{array}{l}\text { Solidez a la } \\
\text { transpiración }\end{array}$ & IUF 426 \\
\hline $\begin{array}{l}\text { Resistencia a } \\
\text { la tracción al } \\
\text { alargamiento }\end{array}$ & IUP 6 & $\begin{array}{c}\text { Mín de distensión a la rotura del } \\
\text { cuero 30\% }\end{array}$ \\
\hline $\begin{array}{l}\text { Cenizas sulfatadas } \\
\text { solubles en agua }\end{array}$ & IUC 6 & Max 1,5\% \\
\hline
\end{tabular}




\begin{tabular}{|l|c|c|}
\hline $\begin{array}{l}\text { Permeabilidad al } \\
\text { vapor de agua }\end{array}$ & IUP 15 & Mín $250 \mathrm{mg} / \mathrm{cm}^{2} \mathrm{~h}$ \\
\hline $\begin{array}{l}\text { Mat. Ext. en } \\
\text { cloruro de } \\
\text { metileno }\end{array}$ & IUC 4 & 10\% Mín $250 \mathrm{mg} / \mathrm{cm}^{2} \mathrm{~h}$ \\
\hline $\mathrm{pH}$ & IUC 11 & Mín 3,5 \\
\hline $\begin{array}{l}\text { Solidez a la } \\
\text { migración }\end{array}$ & IUF 442 & Mín 3 \\
\hline $\begin{array}{l}\text { Resistencia al } \\
\text { calor }\end{array}$ & IUP 18 & Mín 3 \\
\hline
\end{tabular}

En el caso de cuero para capellada, se debería agregar una serie de ensayos opcionales: Solidez a agentes de limpieza (IUF 430), capacidad de almacenamiento (EWG-F1), materias minerales extraíbles (IUC6), etc.

\section{Proceso de Curtido}

Una breve descripción del proceso de curtido se detalla en la figura 1, para el curtido al cromo con la opción de un recurtido cromo o vegetal.

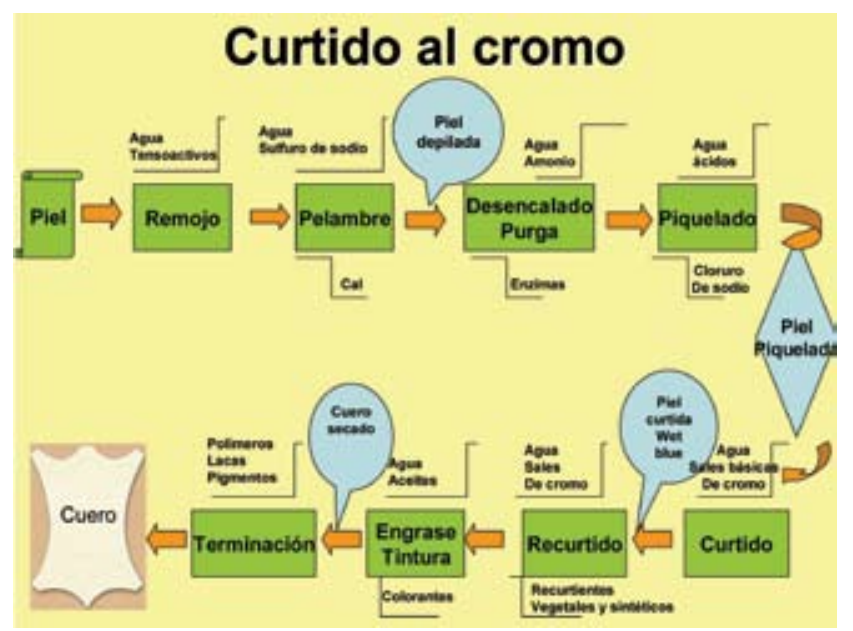

Figura 1 - Diagrama de flujo del proceso de curtido

Este curtido es una formulación convencional, indicándose los tipos de productos a agregar en cada etapa, en nuestro caso haremos un recurtido vegetal con los taninos en estudio.

Para esto trabajaremos en cuero bovino wet blue con un área de 47 p2 $(4,4 \mathrm{~m} 2))$.

Sobre el cuero bovino al estado wet blue se dividió y se trabajó en octavos (ver figura 2).

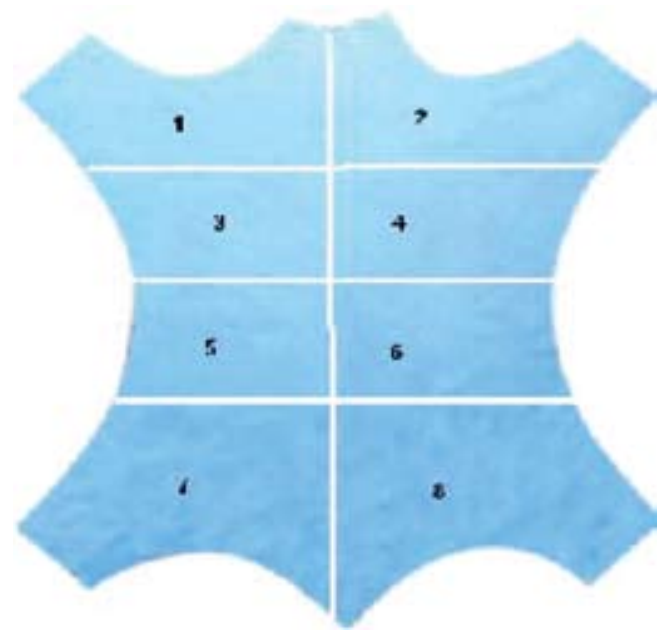

Figura 2 - cuero bovino wet blue
Se analizó, dicho cuero tipificándolo:

\begin{tabular}{|c|c|c|c|}
\hline $\begin{array}{l}\text { Cromo como } \\
\mathrm{Cr}_{2} \mathrm{O}_{3} \text { en base } \\
\text { seca }\end{array}$ & $\begin{array}{c}\text { Cenizas en } \\
\text { base seca }\end{array}$ & $\begin{array}{c}\text { Materias } \\
\text { extraíbles con } \\
\text { diclorometano } \\
\text { (en base seca) }\end{array}$ & $\mathrm{pH}$ \\
\hline $4,1 \%$ & $5,7 \%$ & $3,1 \%$ & $\mathrm{pH}=3.3 \quad$ Ind. Dif. $=0.6$ \\
\hline
\end{tabular}

Por otro lado se recibieron tres taninos vegetales, Tara, Mimosa y Pino Radiata.

\section{Caracterización del los taninos en estudio}

Producto 1. Polvo de Tara, certificado de análisis M 002/2007

Origen: Transformadora Agrícola SAC (Perú)

$\mathrm{N}^{\circ}$ Batch 07033

$\mathrm{pH}=3.7$

\begin{tabular}{|c|c|c|}
\hline Curtientes (\%) & No Curtientes (\%) & Cenizas (\%) \\
\hline 55,2 & 14,9 & 3,1 \\
\hline
\end{tabular}

Producto 2. Extracto de Pino Radiata, certificado de análisis M 002/2007

Origen: Universidad de Concepción (Chile)

$\mathrm{N}^{\circ}$ Batch 07033,

$\mathrm{pH}=3.7$

\begin{tabular}{|c|c|c|}
\hline Curtientes (\%) & No Curtientes (\%) & Cenizas (\%) \\
\hline 32 & 53 & 1,2 \\
\hline
\end{tabular}

Producto 3. Extracto de Mimosa sulfitado Origen: Tannac (Brasil)

$\mathrm{pH}=4,0-4,5$

\begin{tabular}{|c|c|c|}
\hline Curtientes (\%) & No Curtientes (\%) & Cenizas (\%) \\
\hline 59 & 37 & 3,6 \\
\hline
\end{tabular}

\section{Formulación general para el Recurtido}

Wet blue 1,3-1,5 $\mathrm{mm}$ de espesor

Lavado

$0,50 \%$ no iónico

0,50 Ac. Oxálico

Neutralización

$2,50 \%$ Formiato de sodio

Recurtido

$10,0 \%$ Tanino

$1,50 \%$ Ac. Formico

Engrase $\quad 9 \%$ aceite

Fijación $\quad 2,0 \%$ Ac. Formico

$0,5 \%$ Secuestrante de hierro

\section{Ensayos}

Se realizaron varios ensayos comparativos evaluatorios: 


\begin{tabular}{|c|c|c|c|}
\hline \multirow[t]{2}{*}{ Ensayo } & \multicolumn{3}{|c|}{ Muestra } \\
\hline & Tara & Pino Radiata & Mimosa \\
\hline $\begin{array}{l}\text { Resistencia a la } \\
\text { tracción }\left(\mathrm{N} / \mathrm{mm}^{2}\right)\end{array}$ & 14,9 & 13,5 & 11,8 \\
\hline $\begin{array}{l}\text { Resistencia al } \\
\text { desgarre }(\mathrm{N})\end{array}$ & 53,2 & 60,5 & 51,0 \\
\hline $\begin{array}{l}\text { Distensión de la } \\
\text { rotura de la flor } \\
\text { (Lastometro) }\end{array}$ & 11,8 & 9,2 & 11,0 \\
\hline $\begin{array}{l}\text { Flexión seco } \\
(100000 \text { ciclos })\end{array}$ & Sin alteraciones & Sin alteraciones & Sin alteraciones \\
\hline $\begin{array}{l}\text { Flexión húmeda } \\
\text { (50000 ciclos) }\end{array}$ & quiebres & quiebres & quiebres \\
\hline $\begin{array}{l}\text { Envejecimiento } 168 \\
\text { horas a } 100{ }^{\circ} \mathrm{C} \\
\text { Evaluación según } \\
\text { ISO } 105 \text { A02E } \\
\text { (escala de grises) }\end{array}$ & $2-3$ & 2 & 2 \\
\hline $\begin{array}{l}\text { Solidez a la luz } \\
20 \text { horas }\end{array}$ & 4 & $2-3$ & 2 \\
\hline $\begin{array}{l}\text { Solidez a la luz - } 80 \\
\text { horas }\end{array}$ & $3-4$ & $2-3$ & 2 \\
\hline $\begin{array}{l}\text { Materias extraíbles } \\
\text { con diclorometano } \\
\text { (\% expresado en } \\
\text { base seca) }\end{array}$ & 7,2 & 7,3 & 7,1 \\
\hline $\begin{array}{l}\text { Estabilidad a la } \\
\text { humedad }-24 \text { horas } \\
\text { a } 40^{\circ} \mathrm{C}-95 \% \\
\text { humedad relativa }\end{array}$ & $\begin{array}{l}\text { Sin cambio de } \\
\text { color }\end{array}$ & $\begin{array}{l}\text { Sin cambio de } \\
\text { color }\end{array}$ & $\begin{array}{l}\text { Sin cambio de } \\
\text { color }\end{array}$ \\
\hline
\end{tabular}

A efectos de evaluar el comportamiento de los taninos en presencia de anilina negra se realizo un recurtido comparativo usando $2 \%$ de la misma anilina negra (Carbón Derma BFS). El ensayo se comparo frente a un recurtido cromo. La cantidad de anilina agregada fue deliberadamente poca para mostrar mayor diferencia de intensidades.

El resultado de la medida del color fue:

Sistema de color, Método Cie-Lab 1976

\begin{tabular}{|c|c|c|c|c|c|}
\hline & $\begin{array}{c}\text { Standard } \\
\text { blanco }\end{array}$ & Cromo & Mimosa & Tara & Pino \\
\hline $\begin{array}{c}\text { L } \\
\text { (negro } \\
\text { blanco) }\end{array}$ & 93,1 & 34,2 & 37,9 & 41,6 & 38,9 \\
\hline $\begin{array}{c}\text { A } \\
\text { (rojo } \\
\text { verde) }\end{array}$ & $-1,1$ & $-3,0$ & $-1,4$ & $-2,7$ & 0,0 \\
\hline $\begin{array}{c}\text { B } \\
\text { (azul- } \\
\text { amarillo) }\end{array}$ & 1,3 & $-0,7$ & $-2,4$ & $-3,4$ & 1,9 \\
\hline
\end{tabular}

\section{Discusión de resultados}

La tara fue de los taninos en estudio, quién mostró una mejor solidez a la luz.

De los ensayos físicos realizados, el desgarre fue él que diferencio a los recurtientes evaluados con una diferencia considerable del Pino. La tracción mostro diferencias siendo la tara la que mostro menores valores.

Con respecto a la intensidad del color negro pudimos comprobar que el cuero recurtido con cromo fue el más intenso, mientras que la mimosa y el pino tuvieron un comportamiento semejante y el que mostró la menor intensidad fue la tara.

\section{Conclusiones}

Dudimos comprobar que todos los cueros recurtidos (con tara, mimosa y pino radiata) en el estudio partiendo de wet blue para confección de capelladas, cumplían con las especificaciones preestablecidas (salvo los referidos a la tracción) en los ensayos evaluados. Todos los estudios fueron realizados a escala de laboratorio, lo que justificaría continuar con las evaluaciones, repitiendo los diferentes procesos.

\section{Agradecimientos}

Pedro Paz, Daxilan s.a.

Ignacio Pérez, American Chemical ICSA

Alejandro Morales, Química Oriental s.a.

Eduardo Schmidt, Vilmax

\section{Referencias}

Adzet Adzet, J. M. /et al/. Química técnica de tenería. Barcelona : Igualada, 1985

ASTM International. Disponible en: http://www.astm.org/cgibin/SoftCart. exe/index.shtml?E+mystore

BASF. Pocket Book for the Leather Technologist. 4a ed.

Cueronet. Disponible en: http://www.cueronet.com/

Heidemann, E. Fundamentals of Leather Manufacturing, 1993

IULTCS official methods of analysis. Disponible en: http://www.iultcs.org/ iultcs_methods.asp

Mecanipel. Escuela Alemana de Tenería. 191. Reutlingen

Society of Leather Technologists and Chemists, Official Methods of Analysis. Disponible en: http://www.sltc.org/

Tecnología del Cuero. Centro de Investigación de Tecnología del Cuero. Buenos Aires : CITEC

Zhiwen, Ding. Technology of Leather Manufacture. China Leather \& Footwear Industry Research Institute, 2007 